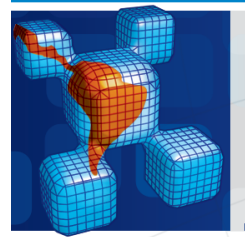

\title{
Numerical simulation of anisotropic polymeric foams
}

\section{Abstract}

This paper shows in detail the modelling of anisotropic polymeric foam under compression and tension loadings, including discussions on isotropic material models and the entire procedure to calibrate the parameters involved. First, specimens of poly(vinyl chloride) (PVC) foam were investigated through experimental analyses in order to understand the mechanical behavior of this anisotropic material. Then, isotropic material models available in the commercial software Abaqus ${ }^{\mathrm{TM}}$ were investigated in order to verify their ability to model anisotropic foams and how the parameters involved can influence the results. Due to anisotropy, it is possible to obtain different values for the same parameter in the calibration process. The obtained set of parameters are used to calibrate the model according to the application of the structure. The models investigated showed minor and major limitations to simulate the mechanical behavior of anisotropic PVC foams under compression, tension and multi-axial loadings. Results show that the calibration process and the choice of the material model applied to the polymeric foam can provide good quantitative results and save project time. Results also indicate what kind and order of error one will get if certain choices are made throughout the modelling process. Finally, even though the developed calibration procedure is applied to specific PVC foam, it still outlines a very broad drill to analyze other anisotropic cellular materials.

\section{Keywords}

Polymeric foams; Anisotropy; Parameters calibration; Material models.

\section{Volnei Tita* and Mauricio Fran- cisco Caliri Júnior}

Aeronautical Engineering Department, Engineering School of São Carlos, University of São Paulo Av. Trabalhador São-Carlense 400, São Carlos, SP, Brazil

Received 29 Mar 2012;

In revised form 28 Apr 2012

* Author email: voltita@sc.usp.br

\section{INTRODUCTION}

Structural modelling of polymeric foams is an intricate task, especially when the cellular material involved presents well defined elastic and plastic anisotropic mechanical behaviors. This intricate task occurs for example on dynamic and quasi-static structural analyses for sandwich composite airplane structures, where the skins are made from composite material and the 
core is made from polymeric foams. In this case, not only the skins, but also the core shows anisotropic effects under multi-axial loadings, which influence the structure's response. In fact, the manufacturing process of cellular materials, especially polymeric foams, might greatly influence its phenomenological mechanical response, which is composed by the contributions from the micro structures (micro cells) within the material. Gong, Kyriakides and Jang[11] showed how the micro structure of the cells influence the structural strength and stability of a polymeric foam. Also, a broad data about manufacturing processes of polymeric foams provides a lot of insights about the micro structure's final shape [13]. Thus, an anisotropic mechanical behavior is not a very uncommon characteristic found in foams. The micro mechanisms of failure, which occurs in these foams, play an important role in the hardening process, because the mechanisms can indicate brittle, plastic or perfect plastic response of the material after its elastic regime and according to the type of loading applied. Besides, these materials present great strain energy potentials, which make them attractive to structural impact applications. Their low relative densities (the ratio of the cellular material density to the base material density) turn these materials very strategic to be applied on thermal and acoustic isolation as well. These particularities and other reasons have motivated many researchers into investigating cellular materials. Gibson and Ashby [8]wrote a landmark book and, besides, a whole micromechanical approach for cellular materials, several potential applications can be found. Newer and more common applications for cellular structures comprise the use of sandwich structures. For example, the special foams used in modern structures such as aircrafts and boat hulls. Thus, a lot of scientific works can be seen in the literature about the mechanical behavior prediction of structures made from cellular materials, using analytic and numerical analyses combined to experimental tests $[10,15,17-20,22,26]$. However, many works do not consider the anisotropic effects and the numeric simulations are carried out using only the input data (material model parameters and hardening curve) from uniaxial compression test for one anisotropy direction[18, 19].

To master the modelling of such structures and materials, one must go over theories of elasticity and plasticity to properly manage yield surfaces and hardening laws[24]. Adding numerical issues from non linear FEA (Finite Element Analyses) to these different elasticplastic behaviors makes the modelling of anisotropic responses a non trivial task. Engineers and designers often use implemented material models in FEA commercial codes to save time during the project development. If the particular material model available in the FEA software does not yield representative results, another material model can be implemented via a user sub-routine, but this approach depends on the FEA software resources. Furthermore, a yield surface, which accounts for different strengths in compression and tension, as well as different hardening intensities that vary with the loading path and the material direction, is rarely found and properly used. In addition, the models must be capable of dealing with large logarithmic strains (higher than 100\%). Therefore, it is very important to carefully select and use material models implemented in FEA commercial codes, but of utmost importance is the calibration of the material model parameters. The main challenge is how to properly set parameters values to an isotropic material model in order to simulate the mechanical behavior 
of the anisotropic cellular material. It must be also mentioned that the inherent numeric issues from the assumptions made in the implemented material model can add up in the simulations rendering large errors. All these aspects are rarely discussed in the literature, because most of the contributions focus on assuming that the cellular materials is isotropic $[18,19]$ or focus on developing a new anisotropic material model $[5-7,9,25,27]$ without concerning whether or not such model is worthy applied. Isotropic material models are very important, but often, the anisotropy degree of cellular materials is very high to be neglected without any insights into the material's micromechanics. On the other hand, anisotropic material models have a huge number of parameters to be identified (usually, over ten different types of experimental tests are required [25]). Engineers and designers prefer to use isotropic material models in order to predict the mechanical behavior of products made from anisotropic cellular materials (e.g. polymeric foams), because the number of experimental tests to identify parameters does not demand a lot of time, specially, during the conceptual and preliminary design phases. Therefore, it is very important to properly handle the parameters in these material models, using adequate procedures.

Regarding the comments above, this paper shows in detail the modelling of anisotropic polymeric foams under compression and tension loadings, focusing on the numeric issues that influence the results. Also the accuracy of such results is investigated based on the calibrated parameters. Firstly, specimens of poly(vinyl chloride) (PVC) foam, were investigated through experimental analyses in order to understand the mechanical behavior of this anisotropic material[21]. Secondly, by inspecting the experimental results and based on the Abrate's [5] study on material models for cellular structures as well as observing most of the references in the literature, it was decided to work with two material models available in the commercial software Abaqus $^{\mathrm{TM}}$ [1]. They are based on the work developed by Deshpande and Fleck [7] and are judged to yield good results; however, the ability of these models for simulating anisotropic structures should be shown. Naturally, there are advantages and limitations to simulate the mechanical behavior of PVC foam, using these material models, but they can be minimized or even overcome with suitable parameters calibration. Thus, for the material models chosen, a calibration procedure was developed in order to identify the most appropriate parameters and hardening curves through only three or five experimental tests. Even though the calibration procedure is applied for specific PVC foam, it still outlines a very broad drill to analyze other anisotropic cellular materials.

\section{MECHANICAL BEHAVIOR OF ANISOTROPIC POLYMERIC FOAMS}

Closed-cell rigid foam, Divinycell ${ }^{\mathrm{TM}} \mathrm{H} 60$ from DIAB [4], was investigated in this work (Fig. 1). The base material is the poly (vinyl chloride) (PVC) polymer, which has density of $60 \mathrm{~kg} / \mathrm{m}^{3}$ and relative density around $0.043\left(\rho_{\mathrm{PVC}}=1400 \mathrm{~kg} / \mathrm{m}^{3}\right)$. There is also anisotropy in this material, which can be classified as transversely isotropic. Then, two sets of mechanical properties must be determined: in-plane properties (for directions 1 or 2) and out-of-plane properties (for direction 3) (Fig. 1). 


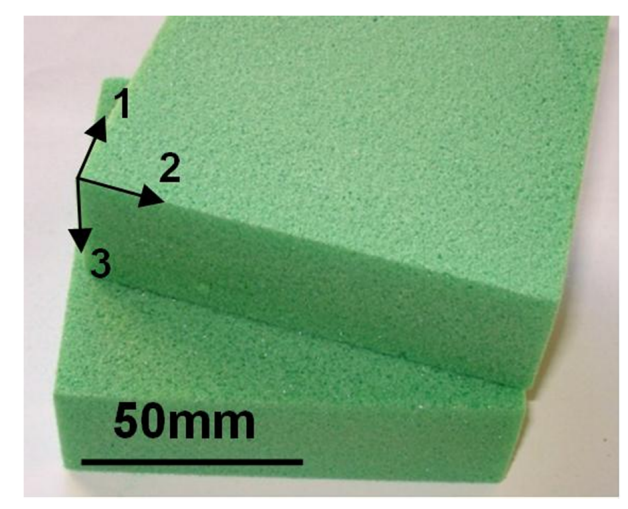

Figure 1 PVC foam: material axes (1-2-3). Transversely isotropic material: in-plane directions (1 and 2); out-of-plane direction (3)

The foam's final micro structure and its anisotropic behavior are related according to Fig. 2(a), which shows elongated cells in direction 3, similar to oblongs. Figure 2(b) shows a nearly circular pattern for plane 1-2. Such disposal renders denser transversal planes to planes 1-3, due to the presence of more vertices and edges, as well as thicker micro structures, which stiffs and strengthens the material in the normal direction 3 (out-of-plane direction). This mechanical behavior can be confirmed by Fig. 3, which shows the phenomenological response for PVC foam under uniaxial compression in both directions (out-of plane direction or direction 3 ; in-plane direction or direction 1 or 2 ).

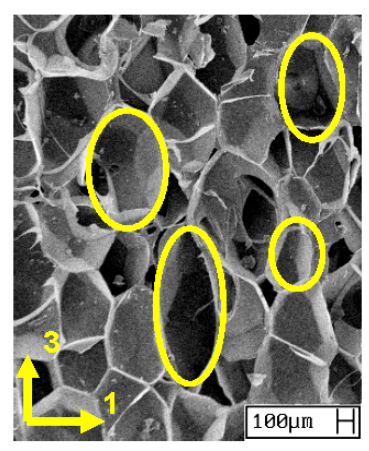

(a)

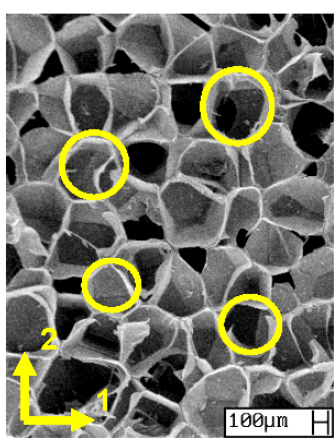

(b)

Figure 2 Micrographs of the PVC foam highlighting the pattern of the cells: (a) plane 1-3; (b) plane 1-2.

Under uniaxial compression loadings, the material exhibits a linear elastic phase as shown in Fig. 3. Past the yield stress, the material behaves similarly to perfect plastic regular materials up to a point where almost all cells have buckled in what is known as crushing due to compression. Then, the densification process occurs with the self contact of the cells in the micro structures, which increases the strength and stiffness foam. Therefore, buckling no longer takes place and the foam, which is a structure, presents a mechanical behavior close to its base material (PVC). Under uniaxial tension loading, contrary to compression, 
the foam presents quasi-brittle response (Fig. 4). The material fails by overall rupture of the structure at approximately $4 \%$ strain, unlike compression, where logarithmic strains over 100\% are observed.

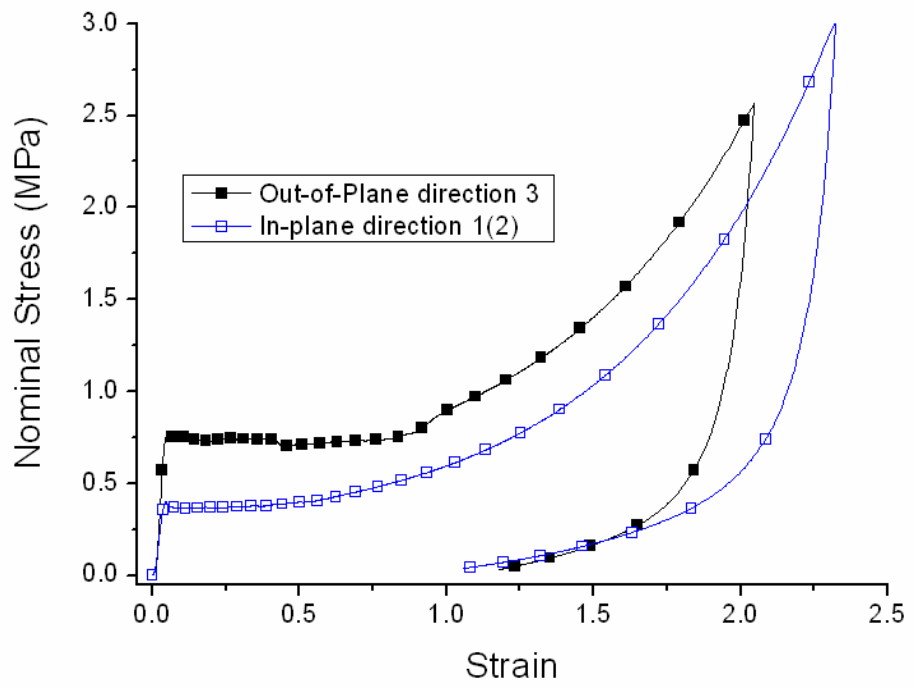

Figure 3 Stress-strain curves for PVC foam under uniaxial compression test.

The failure micro mechanisms such as micro buckling and plastic hinges in the microstructure can be observed under compression loadings at Fig. 5(a). Since the cells are closed, there is also the burst of faces shown at Fig. 5(b). It is important to mention that depending on the relative density of the material and the base material, the failure micro mechanisms and the phenomenological behavior of the foam may change from anisotropic to nearly isotropic and from flexible to brittle [8]. In case of the PVC foam (Divinycell ${ }^{\mathrm{TM}} \mathrm{H} 60$ ), it was verified that the foam is transversely isotropic with flexible behavior under uniaxial compression, but quasi-brittle behavior is observed under uniaxial tension load. More details about the material response can be seen at another publication performed by the authors [21].

\section{MATERIAL MODELS}

The material models were selected considering all the phenomenological responses of the PVC foam. As observed in the experimental tests $[2,3]$, different behaviors under compression and tension loadings, added to the anisotropy (transversely isotropic), restricting the choices of material models, which must be carefully calibrated to obtain reliable results, especially at large logarithmic strains $(\varepsilon>100 \%)$.

Based on the experimental results, the software Abaqus ${ }^{\mathrm{TM}}$ is chosen to perform the numerical analyses, because this commercial code contains material models implemented for crushable foams based on Deshpande and Fleck's work [7]. In fact, one of the models is an authentic representation of the simplified version of Deshpande and Fleck's model. This material model 


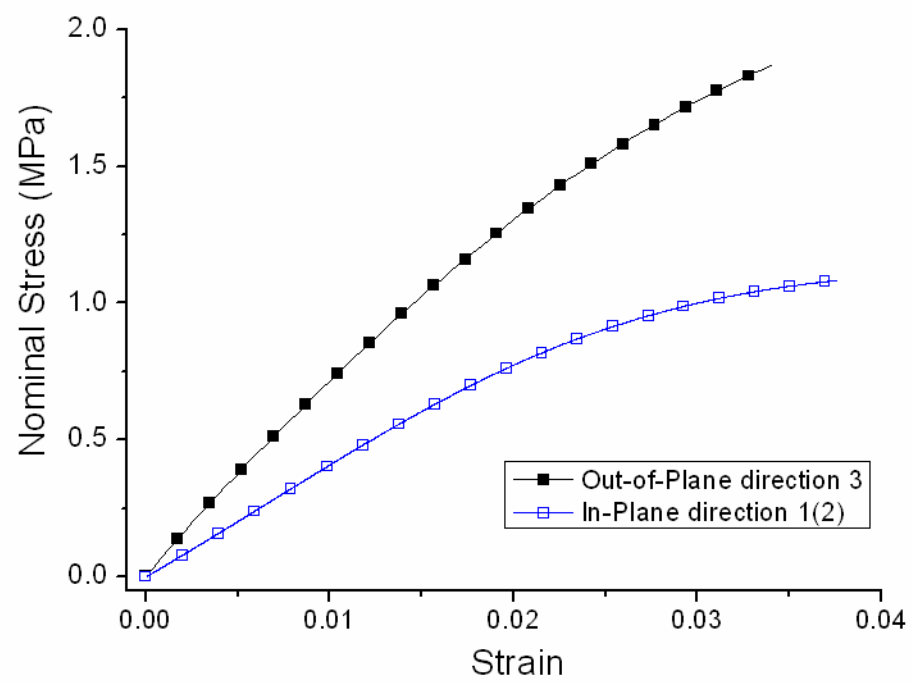

Figure 4 Stress-strain curves for PVC foam under uniaxial tensile test.

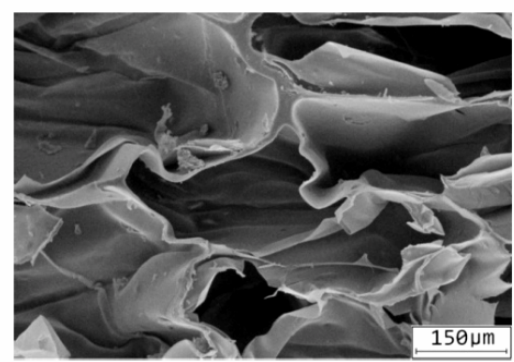

(a)

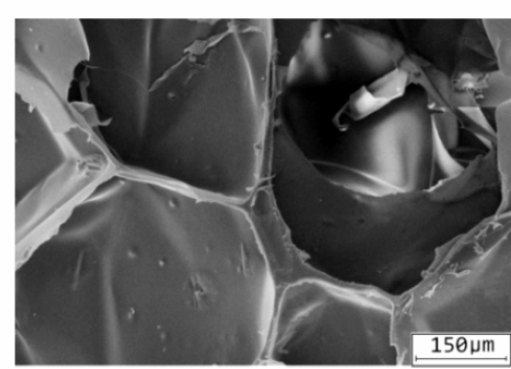

(b)

Figure 5 Failure micro mechanisms of PVC foam: (a) Micro buckling and plastic hinges; (b) Burst cells.

is referred as Crushable foam with Isotropic Hardening (CIH). However, there is also another one, available inside Abaqus ${ }^{\mathrm{TM}}$, which accounts for the brittle response in tension and the high strain energy capacity of foams in compression. This material model is named Crushable Foam with Volumetric Hardening $(\mathrm{CVH})$ [4]. More information about this type of model can be found at Chen [24] and Liu and Carter [16], who discussed material models for soil's such as the Cam Clay model.

The CIH model assumes an isotropic yield surface, as well as an isotropic hardening with control of the flow potential through the plastic Poisson's ratio. As for the CVH material model, it assumes a null plastic Poisson's ratio, hence no control over the flow is available, but there are some parameters through which one can shape the initial yield surface to control the numerical limitations (e.g. convergence problems).

The CVH material model implemented is represented by the yield surface written in Equations 1 and 2:

Latin American Journal of Solids and Structures 1(2012) $1-21$ 


$$
\begin{gathered}
f=\left[\sigma_{v}^{2}+\alpha^{2}\left(\sigma_{m}-\sigma_{0}\right)^{2}\right]-\left[1+(\alpha / 3)^{2}\right] \sigma_{y}^{2} \leq 0 \\
\sigma_{v}=\sqrt{\frac{3}{2} S_{i j} S_{i j}} \text { and } \sigma_{m}=-\frac{\sigma_{k k}}{3}
\end{gathered}
$$

Where $\sigma_{v}$ is the von Mises equivalent stress; $\sigma_{m}$ is the mean stress; $\sigma_{0}, \alpha$ and $\sigma_{y}$ are parameters to be identified experimentally (later detailed in this paper); $S_{i j}$ is the second invariant of stress deviator tensor.

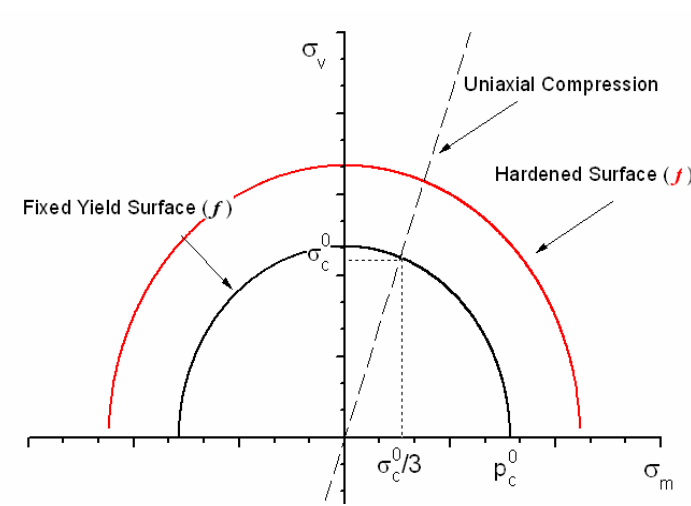

(a)

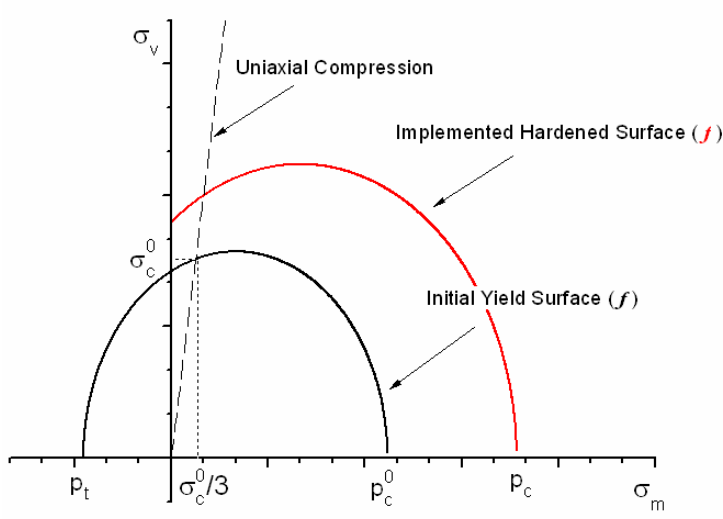

(b)

Figure 6 Initial yield surface: (a) $\mathrm{ClH}$ model (isotropic hardening); (b) CVH model (volumetric hardening).

Figure 6(a) illustrates the yield surface for the CIH model, which evolves uniformly on all loading paths. Figure 6(b) shows the initial yield surface and a new generic yield surface, for the CVH material model. The negative hydrostatic tension yield stress is represented by $p_{t}$; $p_{c}$ corresponds to the positive hydrostatic compressive yield stress (with superscript "zero" corresponds to the yield initial value) and ${\sigma_{c}}^{0}$ corresponds to the uniaxial compression yield stress. By looking at Figure 6(b), for null or negative mean stress values (i.e. for a state of tension hydrostatic stress), the surface does not yield and the material is assumed to be perfect plastic [4]. Thus, the surface does not evolve at the left side of the invariant stress plane $\left(\sigma_{v}\right.$ vs. $\left.\sigma_{m}\right)$. However, when the mean stress values are positive, i.e., compressive loadings occur, the surface normally evolves at the right side of the invariant stress plane.

Such approach is based on the fact that foams usually have a hydrostatic compressive yield stress much larger than the correspondent hydrostatic tension yield stress. The guidelines in Abaqus $^{\mathrm{TM}}$ [1] suggest that this limit in tension is around 5 to $10 \%$ of the compression one. In these cases, there is a small "gap" between the original and the hardened yield surfaces under tension and pure shear loadings that can be negligible without expecting considerable numerical issues. Furthermore, crushable materials usually show perfect plastic behavior up to large logarithmic strains (higher than 50\%). Then, the original yield surface does not abruptly evolve and the discontinuity is kept at a minimum or even eliminated. These assumptions must be 
carefully followed mainly for tension and shearing loads. If the cellular material presents strong hardening and if the hydrostatic tension yield stress is higher than the $5-10 \%$ of the compressive yield stress for the respective yield surface, then, under multi-axial loadings (shearing combined with tension loadings), numerical issues will occur. These errors are expected due to the discontinuity in the yield surface, which cannot be neglected anymore. Managing element size, shape functions and mesh density may control the convergence of the numerical solution to possibly circumvent or overcome such limitation of the implemented material model. On the other hand, analyses under mainly compressive loadings are represented accurately.

Finally, based on the formulation shown, despite all limitations, the authors believe that the CVH model can better simulate the mechanical behavior of the PVC foam herein discussed. Mainly, because the CVH model offers more control over the initial yield surface, which allows one to better represent the elastic and plastic anisotropic behavior of the cellular material. Hence, in this work, focus will be given to the CVH model rather than the $\mathrm{CIH}$ one, which poses no great modelling issues. However, to properly use the CVH material model, its parameters must be calibrated carefully and according to the application of structure in service. For this paper, the difficulties in calibrating the CVH model to better simulate the anisotropic rigid PVC foam are discussed and how accurate and trustful the results are.

\section{PARAMETERS CALIBRATION PROCEDURE}

Regarding the material models described earlier, it is not so difficult to identify $\sigma_{y}$ and $\sigma_{0}$. First, the material's yield stress $\left(\sigma_{y}\right)$ is evaluated using uniaxial tests. Second, $\sigma_{0}$ is the translation value of the yield stress in the mean stress axis (value at the abscissa coordinate). However, the parameter $\alpha$ is the most intricate one that it is defined by Equations (3-5), which show its relation to the shape of the yield surface [4]:

$$
\alpha=3 k / \sqrt{\left(3 k_{t}+k\right)(3-k)}
$$

$$
k=\sigma_{c}^{0} / p_{c}^{0}
$$$$
k_{t}=p_{t} / p_{c}^{0}
$$

Thus, parameter $\alpha$ depends on the $k$ and $k_{t}$, which are the actual parameters to be calibrated within Abaqus ${ }^{\mathrm{TM}}$ along with the hardening curve. As for the elastic phase, the material is considered isotropic; so the elastic Poisson's ratio and Young's modulus are required as usual. In the plastic regime, the $\mathrm{CVH}$ model assumes a null plastic Poisson's ratio $\left(v_{p l}\right)$, which locks the control over the flow potential. A control over the shape of the yield surfaces through the ratio $k$ and $k_{t}$ are offered instead. An initial isotropic yield surface can be defined in the CVH model by setting $\sigma_{0}$ equal to "0" (zero) and $k_{t}$ equal to "1" (one).

As for the CIH material model, it is only necessary to define the $k$ ratio value, but now the plastic Poisson's ratio is required. Changing the plastic Poisson's ratio allows a better control over the hardening as to the hydrostatic and shear stress contributions in the plastic regime, even though this control is done symmetrically to the shear stress axis. Despite the fact the

Latin American Journal of Solids and Structures 1(2012) $1-21$ 
CVH locks control over the plastic Poisson's ratio; it provides an anisotropy control with the introduction of the parameter $k_{t}$.

Definitions of $k$ and $k_{t}$ parameters must be well understood and the consequences of their numeric values must be investigated. The latter is often overlooked. The first one is the quotient of the initial yield stress in uniaxial compression $\left(\sigma_{c}{ }^{0}\right)$ to the initial yield stress in hydrostatic compression $\left(p_{c}{ }^{0}\right)$ as shown by Eq. 4. The second one is the quotient of the yield stress in hydrostatic tension $\left(p_{t}\right)$ to the initial yield stress in hydrostatic compression $\left(p_{c}{ }^{0}\right)$ as shown by Eq. 5. The hydrostatic tension does not carry the super script " 0 ", because the yield surface does not evolved under null or tensile mean stresses (source of possible discontinuities). Based on $k$ and $k_{t}$ values, Abaqus ${ }^{\mathrm{TM}}$ calculates the current yield surface for each foam material point at the finite element model. To complete the input data set for the $\mathrm{CIH}$ and $\mathrm{CVH}$ material models, a table containing the logarithmic plastic strain and the correspondent Cauchy stress must be provided for the finite element model in order to control the evolution of the yield surface.

The procedure for determination of parameters consists on data from uniaxial compressive and tensile tests, and from hydrostatic compressive tests. Uniaxial tests are usually easy to perform, but hydrostatic tests are not. One could replace the hydrostatic test by a shear test to calibrate the third parameter. However, since the material has two sets of properties, choosing a plane (1-2 or 1-3(2-3)) to perform the shear test may be questionable as to which one better suits the current analysis; given that one is dealing with multi-axial loadings. Moreover, the cellular characteristic of the material implies different failure mechanisms depending on the loading path. Hence, due to these issues, choosing the hydrostatic compression test not only improves the identification of the parameters towards an isotropic value for the anisotropic foam, but also in regard to its failure mechanism in compressive loading.

The uniaxial tests (compression and tension) were taken for both planes (1-2 and 1-3) with loading in both directions (1(or 2) and 3). Most of experimental tests followed standards tests $[2,3]$ and were supported by a Digital Image Correlation (DIC) technique [12, 21]. In this work, data for directions 3 and 1 were evaluated to study the anisotropic behavior of the PVC foam. The yield stress in uniaxial compression was set to be the maximum stress level at the beginning of the plateau level, usually indicated by a peak after the linear elastic regime (Fig. 3). Under uniaxial tension, due to the quasi-brittle response observed (Fig. 4), a yield stress for the foam was set to occur at $1 \%$ of total strain in this work. This assumption is helpful in the calibration of the material model, which requires a priori, values of yield stress, not strength values. In the case of the hydrostatic test, the yield stress was identified when a large volumetric strain began to take place in the sample with a reduction of the total pressure shown by the hydrostatic compression system. These tests provided the necessary data to determine different initial yield surfaces, from which the parameters $k$ and $k_{t}$ are calculated, as well as different hardening curves. It is important to mention that if the CIH material model is chosen, then only three experimental tests are required: uniaxial compression (Fig. 7(a)) or tension tests (Fig. 7(b)) for both material planes (1-2 and 1-3) and the hydrostatic compression test (Fig. 7(c)). 


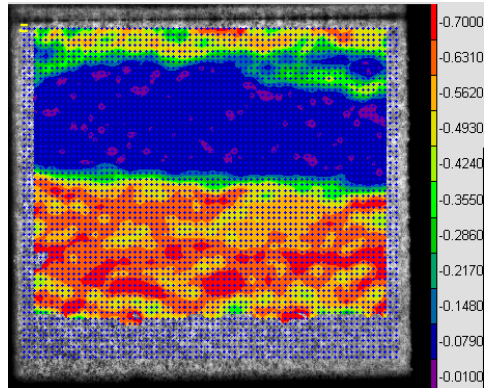

(a)

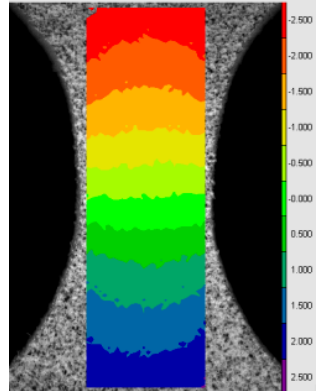

(b)

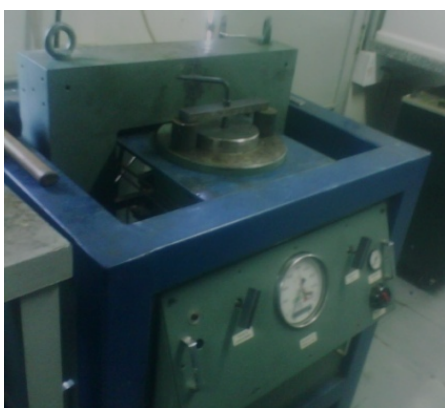

(c)

Figure 7 Experimental tests required: (a) Specimen under uniaxial compression (DIC measurements); (b) Specimen under uniaxial tension (DIC measurements); (c) Hydrostatic compression equipment.

Therefore, based on only three $(\mathrm{CIH})$ or five $(\mathrm{CVH})$ experimental tests, yield surfaces are built and the parameters can be obtained. However, it is possible to calculate different values for the same parameter, due to the anisotropic response of the material as shown at Table 1. Thus, in this work, six different yield surfaces for the PVC foam were built, four surfaces (I, II, V and VI) for the CIH model and another two surfaces for CVH model (III and IV).

Table 1 Parameters obtained for the PVC foam

\begin{tabular}{cccccccc}
\hline Surface/Model & Plane & UCYS (MPa) & UTYS (MPa) & HCYS (MPa) & $\mathrm{k}$ & $v_{p l}$ & $\mathrm{k}_{t}$ \\
\hline I/CIH & $1-3$ & 0.750 & 0.750 & 0.450 & 1.6656 & 0.04 & - \\
II/CIH & $1-2$ & 0.396 & 0.396 & 0.450 & 0.8801 & 0.02 & - \\
III/CVH & $1-3$ & 0.750 & $1.960^{(*)}$ & 0.450 & 1.8852 & - & 12.2965 \\
IV/CVH & $1-2$ & 0.396 & $1.070^{(*)}$ & 0.450 & 1.9522 & - & 5.1478 \\
V/CIH & $1-3$ & 0.714 & 0.714 & 0.450 & 1.5832 & 0.04 & - \\
VI/CIH & $1-2$ & 0.430 & 0.430 & 0.450 & 0.9540 & 0.49 & - \\
\hline \multicolumn{2}{c}{ UCYS: Uniaxial Compression Yield Stress; UTYS: Uniaxial Tension Yield Stress; }
\end{tabular}
HCYS: Hydrostatic Compression Yield Stress; $(*)$ Tensile Strength Value.

The next step of the calibrating process consists on defining the "best" yield surface and on identifying the hardening curve adequate in order to simulate the mechanical behavior of the PVC foam. The hardening in the CIH model is simply dictated by a tabular curve of the logarithmic plastic strain versus the respective stress from the experimental uniaxial test. On the other hand, for the CVH model, the hardening input data is more complicated. It is dictated by the stretching of the yield surface in the mean stress axis by the increase of the current hydrostatic yield stress in compression, $p_{c}$ (equation (6)).

$$
p_{c}\left(\varepsilon_{v o l}^{p l}\right)=\sigma_{y}\left(\varepsilon_{v o l}^{p l}\right)\left[\sigma_{y}\left(\varepsilon_{v o l}^{p l}\right)\left(\frac{1}{\alpha^{2}}+\frac{1}{9}\right)+\frac{p_{t}}{3}\right] /\left\{p_{t}+\left[\sigma_{y}\left(\varepsilon_{v o l}^{p l}\right) / 3\right]\right\}
$$

The evolution of the yield surface for the CVH model considers no hardening for null or tensile hydrostatic and mean stresses; so, this is simulated by fixing the value of $p_{t}$ throughout the hardening process. Thus, the surface evolves according to the volumetric compression 
stress $\left(p_{c}\right)$ in function of volumetric plastic strain $\varepsilon_{v o l}^{p l}$. Equation 6 is obtained by applying Eq. 1 to a uniaxial compression test and isolating $p_{c}$. Such procedure allows for the calibration of the hardening curve with a uniaxial compression curve. Based on experimental results for foams, assuming a null plastic Poisson's ratio is physically consistent [8, 10, 11, 14, 23]. For these cases, the total plastic volumetric strain in uniaxial compression equals the axial plastic strain in the loaded direction. Then, the hardening curve can be defined by setting a table with the uniaxial plastic logarithmic strain versus the associated Cauchy stress as mentioned earlier.

The calibration process ends with the determination of which dataset provides the "best" yield surface based on numerical and experimental analyses. Depending on the limitations of the material model to simulate the mechanical behavior of structures made from PVC foam, the set of parameters for a specific application case might be different for other cases. Therefore, the calibration must be carried out according to the application of the foam. Thus, in this work, the determination of the dataset was based on regular case studies. For the first case study, it was considered that the product made from PVC foam is loaded mainly under uniaxial compression, such as helmets. For the second case, the product is to be loaded mainly under tension loadings.

\section{CASE STUDIES}

\subsection{Case Study 1: Uniaxial Compression Loadings}

For this case study, it is considered that the product made from PVC foam would be loaded mainly uniaxial compression loadings. The finite element model had a two-dimensional symmetry (Fig. 8) with boundary conditions at the bottom face and loadings (prescribed displacements at the top face), representing the experimental tests. Plane strain quadrilateral elements with quadratic interpolation (element CPE8 of Abaqus ${ }^{\mathrm{TM}}$ ) were chosen for this case study. Non linear effects were expected due to the large displacements and strains (over 100\%). Furthermore, it is important to mention that the storage matrix was set to unsymmetrical due to the non associative flow of the material models, increasing thereby the computational time. However, uniaxial compressive loading poses no restrictions in respect to the discontinuity in the yield surface.

Each tested direction generated two hardening curves. One curve accounts the spring back phenomenon in the compressed foam and the other one does not. There is a large elastic return of the foam due to micro buckling in the material, which establishes the spring back phenomenon (Fig. 9(a)). As the stress increases, the weakest section fails by rupture of the cells (edges and faces) and there is an associated generation of plastic hinges due to micro buckling of the cells. Once most of cells have buckled, the strength and stiffness of the cellular material increase abruptly with the self contact of cells and the results observed approach the response of the base material, the PVC polymer. If the loadings are removed, the damaged cells exhibit a spring back phenomenon, which influences the phenomenological hardening curves, since the total elastic strain for this cellular material is higher than expected for a 


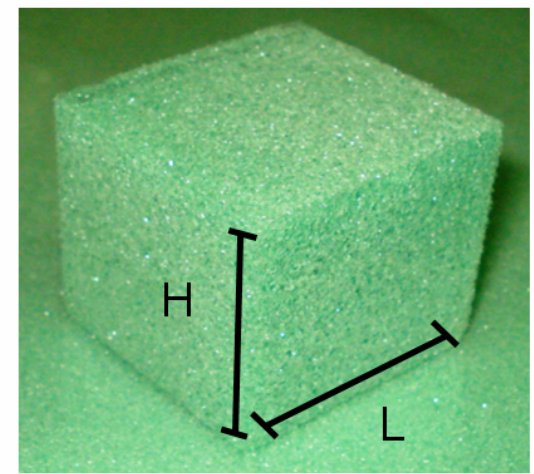

Plane 1-3: $\mathrm{H}=37.5 \mathrm{~mm} ; \mathrm{L}=40 \mathrm{~mm}$ Plane 1-2: $\mathrm{H}=35 \mathrm{~mm} ; \mathrm{L}=37 \mathrm{~mm}$

(a)

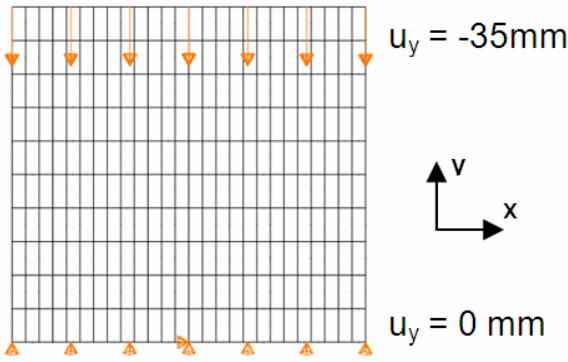

(b)

Figure 8 Case study 1: (a) geometry; (b) mesh and displacements.

regular continuum solid material. Therefore, the total elastic strain $\left(\varepsilon_{\mathrm{C}^{-}}-\varepsilon_{\mathrm{A}}=\varepsilon^{\mathrm{el}}\right.$ Total $)$ can be evaluated by a contribution from the elastic macro response $\left(\varepsilon_{\mathrm{C}^{-}} \varepsilon_{\mathrm{B}}\right)$ and from the portion related to the spring back due to the buckled micro structure $\left(\varepsilon_{\mathrm{B}^{-}}-\varepsilon_{\mathrm{A}}\right)$, as shown in Fig. 9(a). Hardening curves only take inelastic strain into account. Hence, by incorporating the spring back phenomenon, the strain energy absorption of the material is underestimated, because the hardening increases. If the spring back is not taken into account, the energy absorption capacity of the material is properly simulated, and this curve is named "Theoretical Hardening" (Fig. 9(b)). However, modelling the unloading is no longer accurate due to the spring back phenomenon and the phenomenological viscous behavior; so, both phenomena are neglected. This curve is herein named "Real Hardening" (Fig. 9(b)) and the Cauchy stresses are assumed to be equivalent to Nominal stresses, because, under tension loading, the material fails at low strains; hence the increase in the stress was neglected. As for the compression test, the plastic Poisson rules most of the material response and it was verified experimentally a null value for this parameter. Under uniaxial compression, both material models (CVH and $\mathrm{CIH})$ exhibit equivalent responses with no particular notes required. The model with isotropic hardening $(\mathrm{CIH})$ is easier to calibrate since it requires data only from 3 (three) experimental tests, not 5 (five), as in the case of the model with volumetric hardening (CVH).

Figures 10 and 11 portrait the model parameters and show the comparison between experimental results and numerical simulations for the uniaxial compression tests. It is important to mention that the parameters were obtained according to the procedure described earlier. Based on the experimental results, it was possible to determine the respective yield surfaces and hardening curves. As seen in the figures 10 and 11, the "Real Hardening", indicated by curve number 2, assumes that the spring back is taken into account and smaller inelastic strains produce smaller strain energy absorption prior the densification of the material. Meanwhile, the "Theoretical Hardening" (in curves number 3 and 4) smoothly suits the experimental 


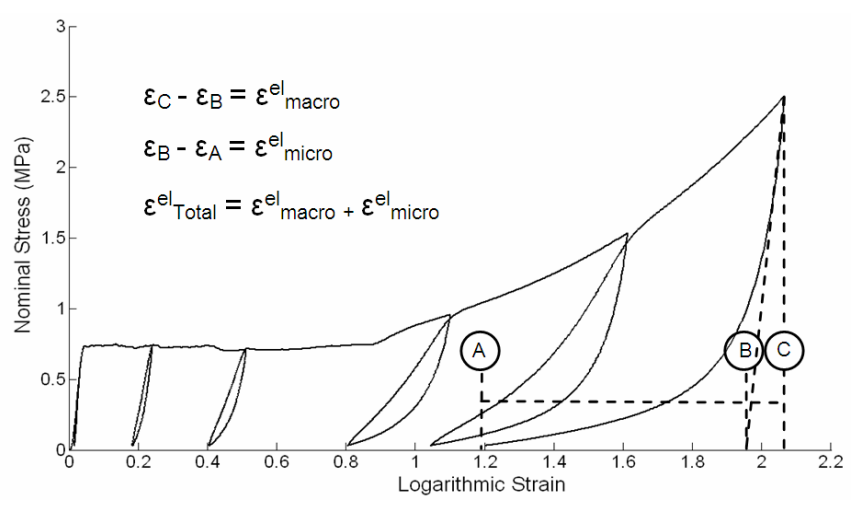

(a)

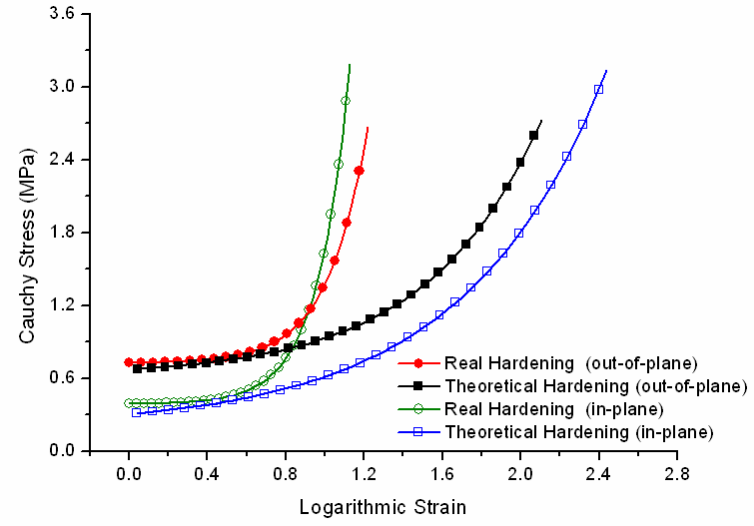

(b)

Figure 9 Uniaxial compression: (a) out-of plane (direction 3) cyclic tests; (b) theoretical and real hardening curves.

curves, because the elastic strain originated in the buckling of the micro cells is incorporated in the total inelastic strain. Then, under compression loading conditions, the elastic strain from the spring back phenomenon should be combined with the total inelastic strain for unloading conditions; the spring back strain should be carefully handled. It is important to observe that the yield surfaces III (CVH model) and I (CIH model) were used to simulate the material response in direction 3. To simulate the material response in direction 1 (or 2), surfaces IV (CVH model) and II (CIH model) were used instead.

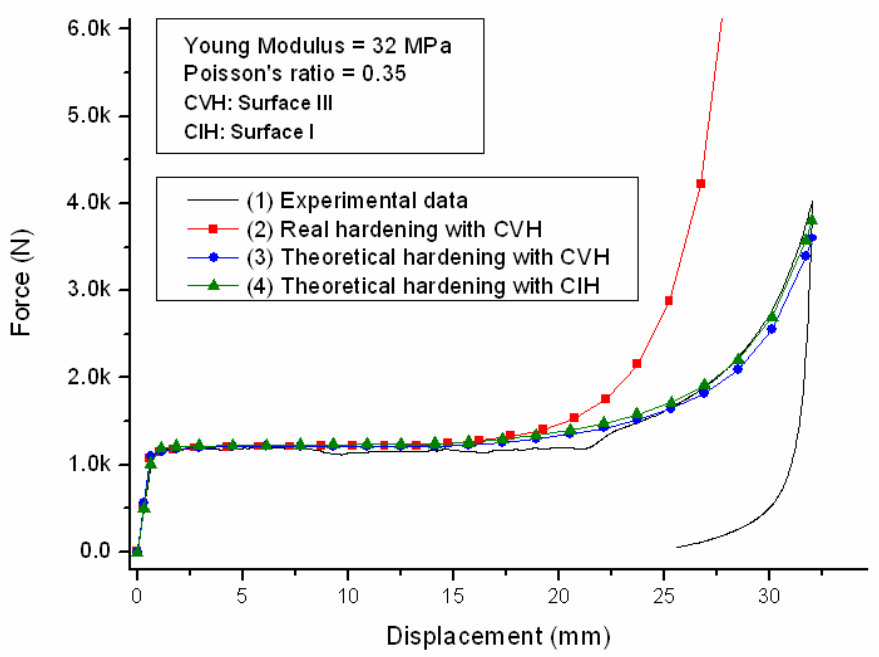

Figure 10 Comparison between experimental and numerical results of uniaxial compression for out-of-plane tests (direction 3).

The null plastic Poisson's ratio effect can be seen in Fig. 12 for the CVH model. Both 


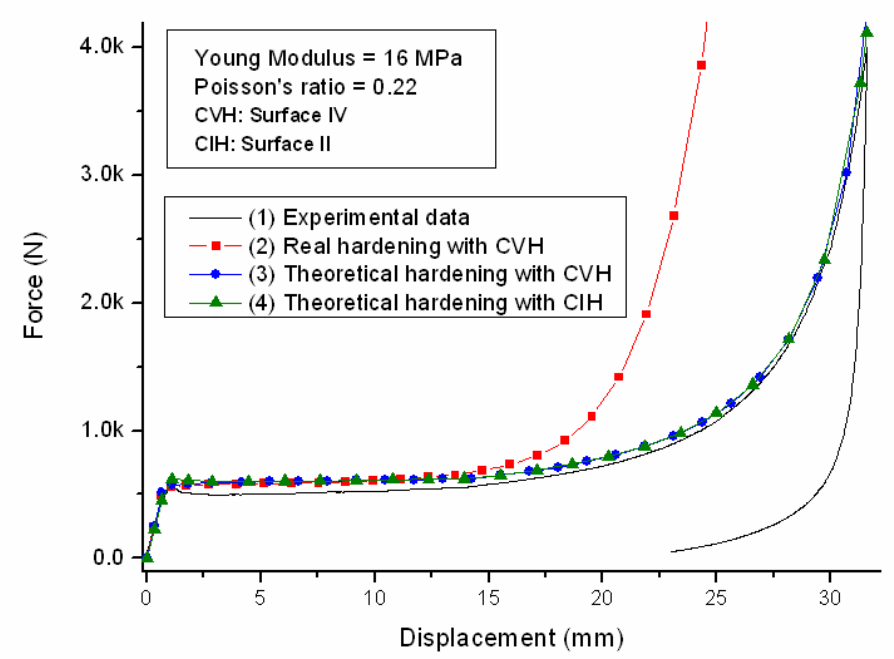

Figure 11 Comparison between experimental and numerical results of uniaxial compression for in-plane tests (direction 1(2))

analyses in direction 3 and 1 (or 2) exhibit a very similar behavior, but the plastic strain shown in Fig. 12 clearly distinguishes the results at the end of the FEA simulation, using the "Theoretical Hardening". At the lower region of the meshes in Fig. 12, a darker and denser mesh is shown and it corresponds to the crushed foam (deformed shape), while the lighter one is the original shape of the material. In Fig. 12(a), the foam crushed in the normal direction 3 has a 192\% logarithmic strain, whereas the foam compressed in the direction 1 (Fig. 12(b)) shows a $233 \%$ logarithmic strain. These numerical results are coherent with the respective strengths, displacement applied and height of specimen used in each simulation.

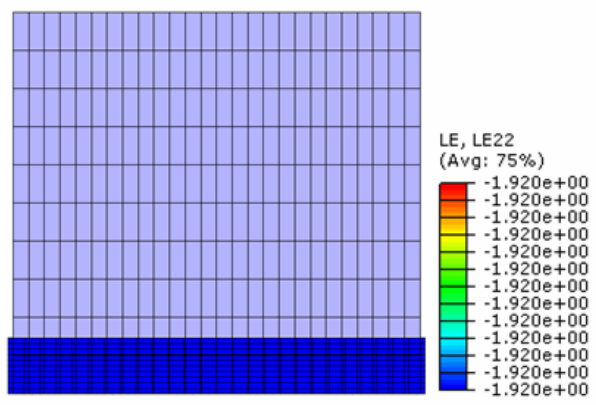

(a)

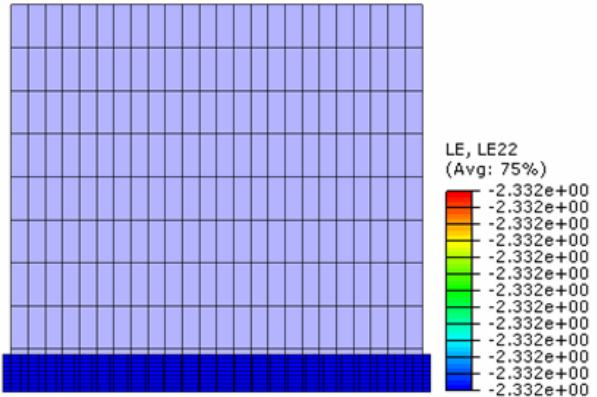

(b)

Figure 12 Effect of the null plastic Poisson's ratio: (a) Logarithmic strain of 1.92 in normal direction 3; (b) Logarithmic strain of 2.33 in direction 1. 


\subsection{Case Study 2: Tensile Loadings}

For this case study, it is considered that the product made from PVC foam is loaded mainly under tension combined to shear. Thus, four different finite element models were developed in order to evaluate the performance of the calibration procedure. In addition for both material models, there were two different geometries to simulate the tension response in direction 3 (outof-plane) and direction 1 (or 2, in plane). Some details about the geometry, mesh, boundary conditions and loadings are shown in Figs 13 and 14. The boundary conditions are applied at the bottom face and loadings are applied at the top face as prescribed displacements. The geometry used in the simulation is based on the format of the foam plates purchased. The thickness of the plate provided by the PVC foam manufacturer restricted the specimens cut for direction 3 to smaller sizes than the ones cut for direction 1 (or 2) (Fig. 13-14). The specimens do not have dimensions to render uniaxial gradient pattern strain in the whole structure, but only at its center. Larger specimens provide larger regions under the uniaxial loading state. Therefore, the parameters identification is carried out based on the experimental results from the center of the specimen obtained by Digital Image Correlation (DIC).

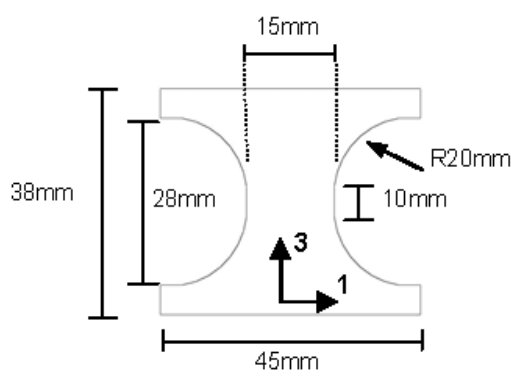

(a)

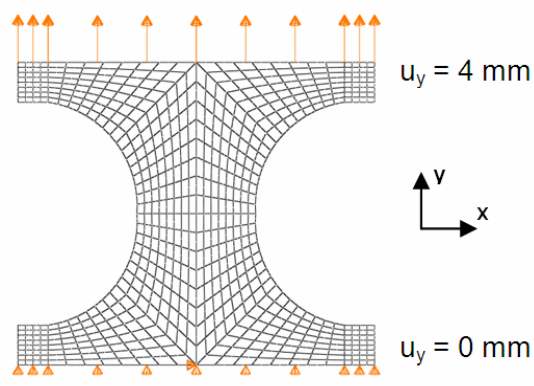

(b)

Figure 13 Case study 2 for out-of-plane tensile test (plane 1-3): (a) geometry draft; (b) mesh and displacements.

The material models behave very differently for tension loadings, i.e., for negative hydrostatic stress contributions. The $\mathrm{CIH}$ material model presents a behavior in tension, which is similar to the hardening response in compression ruled by the tabular input. On the other hand, the CVH material model considers perfect plastic behavior under tension hydrostatic loadings. However, under compression hydrostatic loadings, the material hardens according to the tabular input of the hardening curve and Eq. 6. Hence, there are two approaches for modelling this problem in Abaqus ${ }^{\mathrm{TM}}$. In the first approach, using the volumetric hardening $(\mathrm{CVH})$, the simulation is straightforward, because the implementation considers no hardening. Thus only the initial yield surface is actually used. Different hardening curves may be chosen and the results for the uniaxial tension models will remain unaltered. Often, the hardening curve used with the volumetric model $(\mathrm{CVH})$ is obtained by compression test, which corresponds to the domain where the yield surface does evolve. In the second approach, the isotropic hardening $(\mathrm{CIH})$ can be used, but the material behavior in tension needs to be evaluate with 


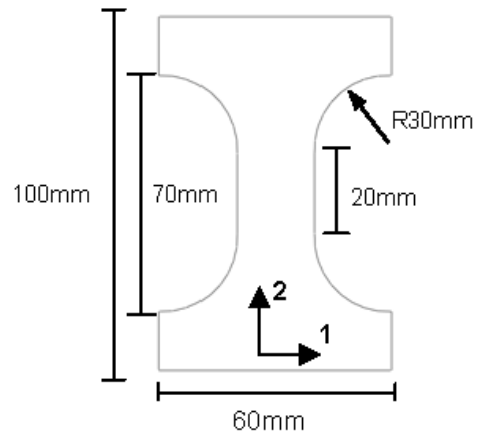

(a)

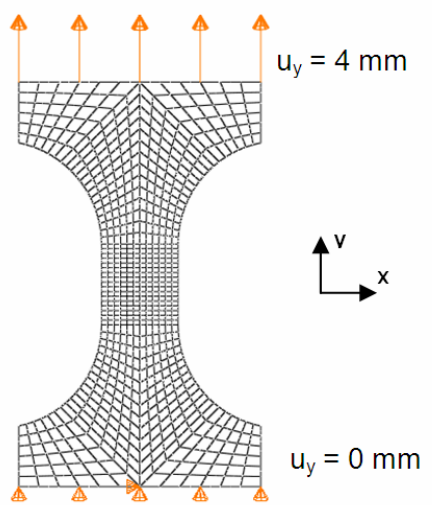

(b)

Figure 14 Case study 2 for in-plane tensile test (plane 1-2):(a) geometry draft; (b) mesh and displacements.

more attention, because the material response is brittle. This may explain the reason why the ASTM standard for polymeric foam under uniaxial tension [3] only handles the material's strength and there are no comments about yield stress. However, when the experimental test was carried out for the PVC foam, it was observed non-linear behavior with inelastic strains (Fig. 15(a)). As commented earlier, in this work, the initial yield stress is considered to take place at $1 \%$ of total strain; so, a subtle hardening may be modeled and these curves can be used for the isotropic material model ( $\mathrm{CIH}$ ) input. Furthermore, the same remarks for compression hardening curves are valid for the tension hardening curves, but micro buckling does not occur in tension loadings and the spring back issues are avoided. Therefore, there is only one hardening curve for each plane of the material and these curves are obtained from cyclic tests (Fig. 15).

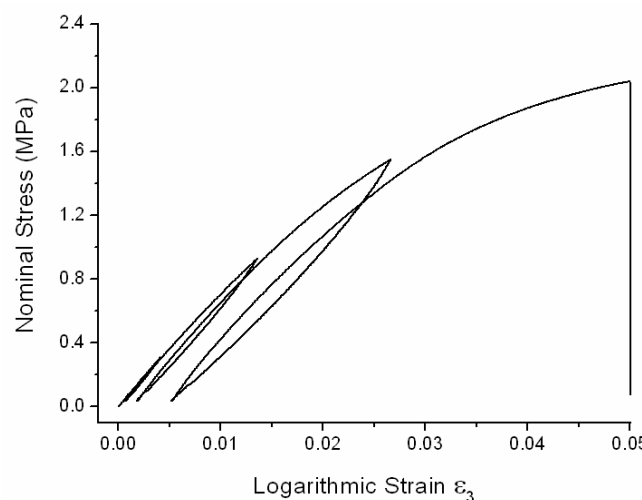

(a)

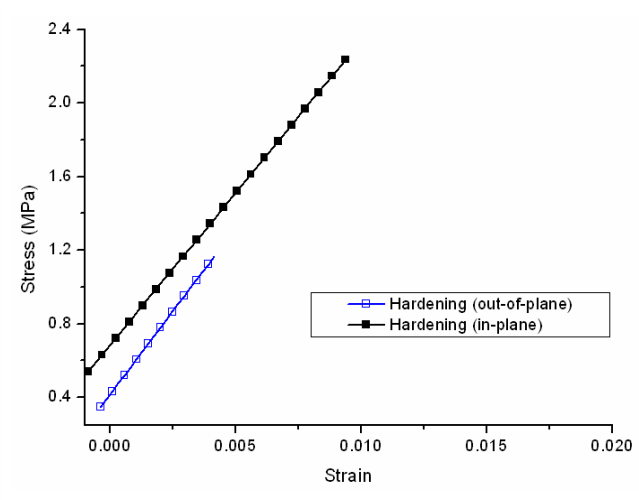

(b)

Figure 15 Uniaxial tension: (a) out-of plane (direction 3) cyclic tests; (b) hardening curves. 
Figures 16 and 17 show the model parameters and the comparison between experimental results and numerical simulations for the tension tests. The parameters were calibrated according to the procedure described, using three or five experimental tests. Based on the tests, it was possible to calculate the respective yield surface and hardening curves, as well as the plastic Poisson's ratio $\left(\nu_{p l}\right)$, which are found at Table 1. It is important to observe that the yield surfaces III ( $\mathrm{CVH}$ model) and V (CIH model) were used to simulate the material response for direction 3 (Fig. 16). To simulate the material response in direction 1 (or 2), surfaces IV (CVH model) and VI (CIH model) were used instead (Fig. 17).

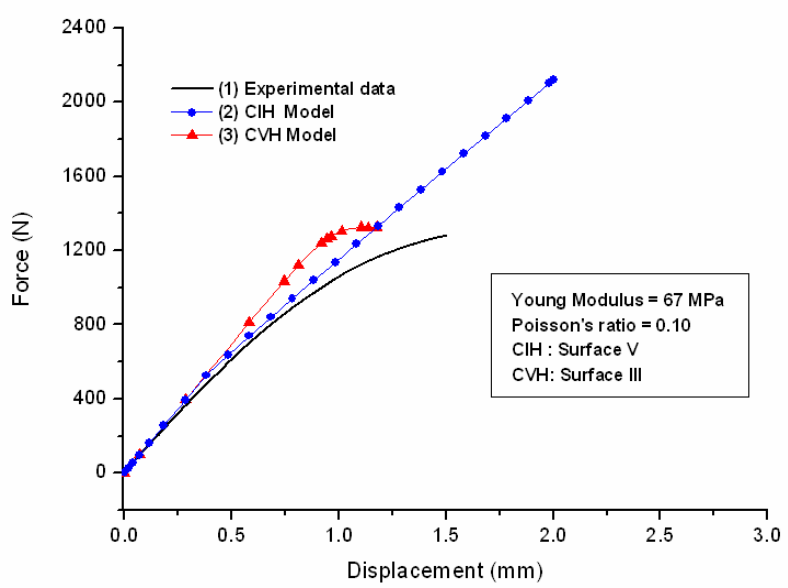

(a)

Figure 16 Comparison between experimental and numerical results of tension for out-of-plane tests (direction 3).

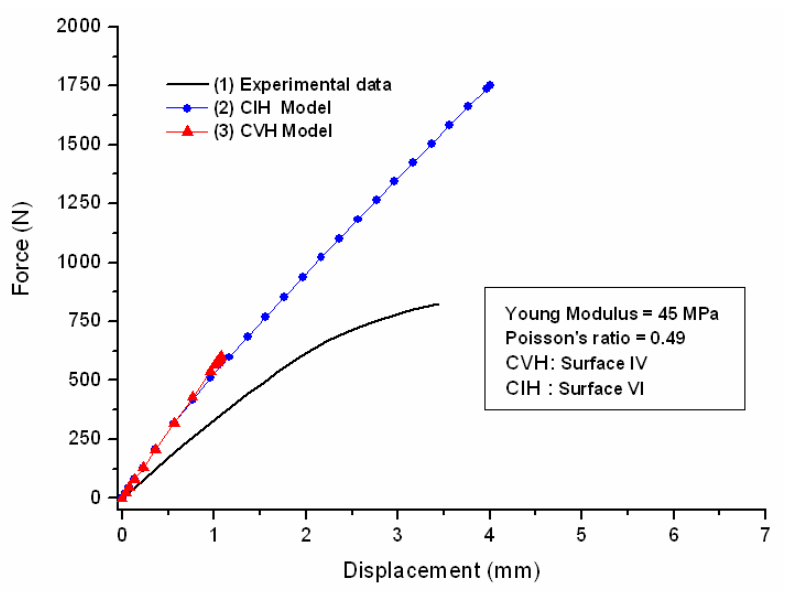

(a)

Figure 17 Comparison between experimental and numerical results of tension for in-plane tests (direction 1(2)). 
By looking at the curve number 3 in Fig. 16, for the tests in direction 3, the CVH model (surface III) assumes perfect plastic behavior, but the analysis terminated prematurely. On Fig. 17, for in-plane tests (direction 1 or 2), one cannot see the difference between material models into the plastic regime, because the analysis using CVH model terminated without converging. More specifically, integration points with null or negative hydrostatic stresses do not exhibit hardening, but those under compression do, as explained earlier. If these material points are in the same element or next to each other, then numerical instability takes place in the results and the numerical analysis fails. To better show this error, from the finite element analysis with results represented by curve 3 in Figure 17, a plot of the hydrostatic pressure with the time increment of the solution is shown in Figure 18 for all 9 integration points of the highlighted element. As the hydrostatic pressure approaches the elastic limit, due to the geometry of the specimen and the loading applied, some integration points unloaded as others are loaded due to the evolution of the multi-axial load. This change in the pattern of the hydrostatic pressure response is naturally sensitive to the time increment size, but it cannot be eliminated. An investigation on the time increment along with fine element size and improved shape function could overcome such discontinuity in the model, but even then the results would oscillate and convergence would not be guaranteed. Moreover, in quasi-static analysis, the convergence for the CVH model in the finite element model cannot depend on element size and time increment. These errors can only be detected for multi-axial loadings, including tension and/or shear. Nevertheless, if a uniaxial test for cubic specimen, similar to those used in the compression tests, is simulated, the results show perfect plastic response and no plastic errors lock the analysis.

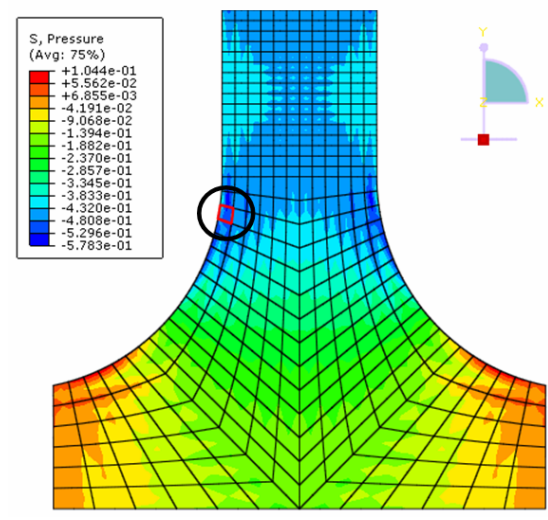

(a)

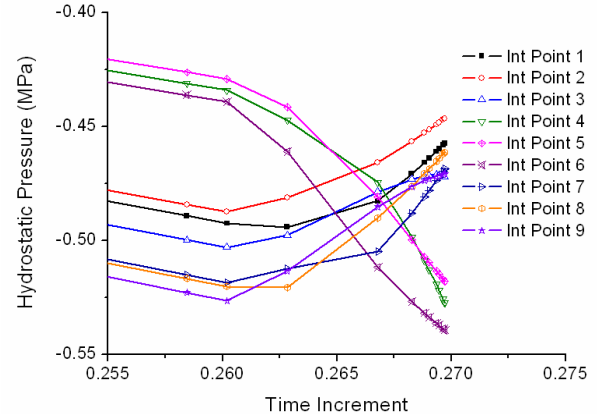

(b)

Figure 18 Numerical results for in-plane tensile test (direction 1 or 2): (a) Hydrostatic pressure and highlighted critical element; (b) Oscillations for hydrostatic pressure in each integration point of the highlighted critical element.

On the other hand, the CIH models (surfaces V and VI) do not present such debilities and the analyses successfully terminate. Even though, the plastic Poisson's ratio for the plane 1-2 is high, the model is fairly short and simple. However it must be emphasized that for the isotropic model, the hardening curve is the same for all loading paths. Therefore, if the 
application is for mainly tension, compression or under multi axial loadings, different curves should be used. Another major difference is found in the results according to the material parameters set used. For the out-of-plane results (direction 3), the CIH models seem to better fit the experimental data, while the CVH model shows a stiffer response up to yielding. Using the in-plane (directions 1 or 2) material set, the numerical models show a much stiffer response than the experimental results (Fig. 17). Such difference is explained by recalling that the material models within Abaqus ${ }^{\mathrm{TM}}$ assume that the foam has an isotropic elastic response, but the PVC foam, as observed, is an anisotropic material. In fact, the anisotropy is close to $50 \%$, i.e., the cellular material is twice stronger and stiffer in direction 3. Hence a more pronounced Poisson's effect is expected, when the material is loaded in direction 1(or 2) since the cross section is now that shown in Fig. 2(a) other than Fig. 2(b).

\section{CONCLUSIONS}

It was verified that the isotropic material models investigated can properly simulate the mechanical behavior of anisotropic foams (e.g. PVC foam) loaded mainly under uniaxial compression. However, it was shown how the shortcut in modelling anisotropic materials with isotropic models is a delicate task to perform. Loading direction and the hardening curves along with the influence of micromechanics effects, like the spring back phenomenon are relevant.

For structures made from anisotropic foams and loaded mainly in tension, the isotropic material models were also able to simulate the mechanical behavior for either direction $1(2)$ or 3. Nevertheless, it was seen that the material model with isotropic hardening can provide better results than with volumetric hardening due to numerical errors. Such errors are due to the multi-axial loadings with comparable (less than 100\% in difference) tension, shear and compression stresses. Therefore, the quality of the quantitative results depends on the loading direction, the type of loading (uniaxial or multi-axial; compression or tension), the hardening model selected and the calibration process of the material model parameters.

Despite the fact that the CVH model performed worse than the CIH in the tension based models, the CVH model is judged a better solution when modelling multi-axial loads in anisotropic foams due to the control over the initial yield surface and different responses according to the loading path. There are more complex models, which avoid such criteria in the calibration process, but other issues appear with the increase of parameters from the models. The developed calibration process and the discussion on the material models herein applied to rigid polymeric foam provide quantitative results for engineers and designers during all project phases. Finally, the results show what kind and order of error one should get if certain choices are made throughout the modelling process.

Acknowledgments The authors would like to thank CNPq (133595/2008-0) for the financial support, and also, Professor Reginaldo Teixeira Coelho for providing a license of software Abaqus $^{\mathrm{TM}}$. Volnei Tita would like to thank the Research Foundation of the State of Sao Paulo (process number: 09/00544-5). 


\section{References}

[1] ABAQUS/CAE User's Manual. ABAQUS version 6.7 - documentation. C Dassault Systemes.

[2] ASTM D 1621 - 04a: Standard test method for compressive properties of rigid cellular plastics. Technical report.

[3] ASTM D 1623 - 03: Standard test method for tensile and tensile adhesion properties of rigid cellular plastics. Technical report.

[4] DIAB Literature - Manuals. Technical Manual: Divinycell H.

[5] S. Abrate. Criteria for yielding or failure of cellular materials. Journal of Sandwich Structures and Materials, 10:5, 2008.

[6] C. Chen, T. J. Lu, and N. A. Fleck. Effect of imperfections on the yielding of two-dimensional foams. Journal of the Mechanics and Physics of Solids, 47:2235, 1999.

[7] V. S. Deshpande and N. A. Fleck. Isotropic constitutive models for metallic foams. Journal of the Mechanics and Physics of Solids, 48:1253, 2000 .

[8] L. J. Gibson and M. Ashby. Cellular solids: structures \& properties. Pergamon Press-Headington Hill Hall, England, 1988.

[9] L. J. Gibson, M. F. Ashby, J. Zhang, and T. C. Triantafillou. Failure surfaces for cellular materials under multiaxial loads -i. modelling. International Journal of Mechanical Sciences, 31(9):635, 1989.

[10] L. Gong and S. Kyriakides. Compressive response of open cell foams part ii: Initiation and evolution of crushing. International Journal of Solids and Structures, 42:1381, 2005.

[11] L. Gong, S. Kyriakides, and W. Y. Jang. Compressive response of open-cell foams. International Journal of Solids and Structures, 42:1355, 2005.

[12] F. Hild and S. Roux. Correli Q4: A software for "finite-element" displacement field measurements by digital image correlation. Technical report, LMT-Cachan.

[13] http://www.poliuretanos.net. Chemistry and polyurethane technology, jan. 5th 2010.

[14] Q. M. Li and R. A. W. Mines. Strain measures for rigid crushable foam in uniaxial compression. Strain, 38:132, 2002.

[15] Q. M. Li, R. A. W. Mines, and R. S. Birch. The crush behaviour of rohacell-51wf structural foam. International Journal of Solids and Structures, 37:6321, 2000.

[16] M. D. Liu and J. P. Carter. On the volumetric deformation of reconstituted soils. International Journal for Numerical and Analytical Methods in Geomechanics, 24:101, 2000.

[17] G. P. Oliveira, R. A. Angélico, V. Tita, and N. C. Santos. An investigation of material model parameters for foams of composite sandwich structures. In V National Congress of Mechanical Engineering - CONEM, Salvador, Brazil. Proceedings of CONEM, 2008.

[18] V. I. Rizov. Elasticplastic response of structural foams subjected to localized static loads. Materials and Design, $27: 947,2006$.

[19] V. I. Rizov. Non-linear indentation behavior of foam core sandwich composite materials - a 2d approach. Computational Materials Science, 35:107, 2006.

[20] V. L. Tagarielli, N. A. Fleck, and V. S. Deshpande. Collapse of clamped and simply supported composite sandwich beams in three-point bending. Composites: part B, 35:523, 2004.

[21] M. F. Caliri Jr.and V. Tita, R. A. Angélico, R. B. Canto, and G. P. Soares. Study of an anisotropic polymeric cellular material under compression loading. In XIX Brazilian Conference on Material Science and Engineering CBECIMAT, Campos do Jordão. Brazil. Proceedings of CBECIMAT, 2010.

[22] T. C. Triantafillou, J. Zhang, T. L. Shercliff, L. J. Gibson, and M. F. Ashby. Failure surfaces for cellular materials under multiaxial loads -ii. comparison of models with experiment. International Journal of Mechanical Sciences, 31(9):665, 1989.

[23] P. Viot. Hydrostatic compression on polypropylene foam. International Journal of Impact Engineering, 36:975, 2009.

Latin American Journal of Solids and Structures 1(2012) $1-21$ 
[24] D. J. Han W. F. Chen. Elasticplastic response of structural foams subjected to localized static loads. Materials and Design, 27:947, 2006.

[25] D. A Wang and J. Pan. A non-quadratic yield function for polymeric foams. International Journal of Plasticity, $22: 434,2006$.

[26] J. Zhang, N. Kikuchi, V. LI, A. Yee, and G. Nusholtz. Constitutive modeling of polymeric foam material subjected to dynamic crash loading. International Journal of Mechanical Sciences, 21(5):665, 1989.

[27] T. Zhang. A general constitutive relation for linear elastic foams. International Journal of Mechanical Sciences, 50:1123, 2008 
